\title{
BMJ Open Depression and blood pressure in high-risk children and adolescents: an investigation using two longitudinal cohorts
}

To cite: Hammerton $\mathrm{G}$, Harold G, Thapar A, et al. Depression and blood pressure in high-risk children and adolescents:

an investigation using two longitudinal cohorts. BMJ Open 2013;3:e003206 doi:10.1136/bmjopen-2013003206

- Prepublication history for this paper is available online. To view these files please visit the journal online (http://dx.doi.org/10.1136/ bmjopen-2013-003206).

Received 10 May 2013 Revised 22 August 2013 Accepted 23 August 2013

Institute of Psychological Medicine and Clinical Neurosciences, MRC Centre for Neuropsychiatric Genetics and Genomics, Cardiff University, Cardiff, UK

Correspondence to Ajay Thapar; ThaparAK@ cardiff.ac.uk

\section{ABSTRACT}

Objective: To examine the relationship between blood pressure and depressive disorder in children and adolescents at high risk for depression.

Design: Multisample longitudinal design including a prospective longitudinal three-wave high-risk study of offspring of parents with recurrent depression and an on-going birth cohort for replication.

Setting: Community-based studies.

Participants: High-risk sample includes 281 families where children were aged 9-17 years at baseline and 10-19 years at the final data point. Replication cohort includes 4830 families where children were aged 1114 years at baseline and 14-17 years at follow-up and a high-risk subsample of 612 offspring with mothers that had reported recurrent depression.

Main outcome measures: The new-onset of Diagnostic and Statistical Manual of Mental Disorder, fourth edition defined depressive disorder in the offspring using established research diagnostic assessments-the Child and Adolescent Psychiatric Assessment in the highrisk sample and the Development and Wellbeing Assessment in the replication sample.

Results: Blood pressure was standardised for age and gender to create SD scores and child's weight was statistically controlled in all analyses. In the high-risk sample, lower systolic blood pressure at wave 1 significantly predicted new-onset depressive disorder in children ( $\mathrm{OR}=0.65,95 \% \mathrm{Cl} 0.44$ to $0.96 ; \mathrm{p}=0.029$ ) but diastolic blood pressure did not. Depressive disorder at wave 1 did not predict systolic blood pressure at wave 3 . A significant association between lower systolic blood pressure and future depression was also found in the replication cohort in the second subset of high-risk children whose mothers had experienced recurrent depression in the past.

Conclusions: Lower systolic blood pressure predicts newonset depressive disorder in the offspring of parents with depression. Further studies are needed to investigate how this association arises.

\section{INTRODUCTION}

The two leading causes of death and disability in the developed world are depression

\section{ARTICLE SUMMARY}

Strengths and limitations of this study

- Replication of findings in two independent cohort studies, one a high-risk sample and the other a population-based cohort.

- In both samples, diagnoses were systematically ascertained using interview and blood pressure readings were measured according to standardised protocol.

- Results need to be replicated in other samples in order to establish a more precise cut-off for low systolic blood pressure.

- Mechanism by which low blood pressure is linked to depression risk needs further investigation.

and cardiovascular disease. The association between depression and cardiovascular disease is well established in adults, ${ }^{1}$ although the mechanisms by which it arises are still not clear. It has been suggested that these links reflect early associations between depression and cardiovascular risk factors. High blood pressure is an important cardiovascular risk factor and it has also been linked to depression in adults in some studies. $^{2}$ Other studies, however, have found the converse. They suggest that depression is associated with low blood pressure and that it is only depression treated with certain antidepressants which is associated with high blood pressure. ${ }^{3}$ To guide the study of mechanisms by which the links between cardiovascular risk factors, notably blood pressure and depression may arise, it is important to first ascertain the direction of effects. In the few longitudinal studies in this area, the findings are inconsistent, with some studies finding depression as a predictor of either high blood pressure ${ }^{4}$ or low blood pressure ${ }^{5}$ while others have found the converse, with low blood pressure predicting depression. ${ }^{6}$

Although depression and the early indicators of cardiovascular disease have been 
found to have the onset in childhood and adolescence, ${ }^{78}$ very few studies have focused on these links in younger populations. In this study, the main aim was to investigate the relationship between blood pressure and subsequent first onset episode of depression in a prospective cohort of children and adolescents at high risk of depression. The secondary aim was to replicate findings in an independent cohort.

\section{METHOD}

Data were derived from a prospective longitudinal study of offspring of parents with recurrent depression-'The Early Prediction of Adolescent Depression (EPAD) Study'. At baseline, the sample included 337 families (315 mothers and 22 fathers) that were recruited from general practices across South Wales, by writing to eligible families and from a database of adults with previously identified unipolar depression. A sample size of 300 families was calculated to provide sufficient power to test the main hypotheses of the original project while allowing detailed assessments at each time point. Recurrent depression is defined as the presence of least two episodes of Diagnostic and Statistical Manual of Mental Disorder, fourth edition (DSM-IV) major depressive disorder. Diagnosis in the index parent was confirmed at baseline using a research diagnostic interview that is widely used to assess adult psychiatric disorder, the Schedules for Clinical Assessment in Neuropsychiatry (SCAN). This was undertaken by researchers who were trained and supervised by an experienced academic clinician. The youngest child within the age range 9-17 years was selected for inclusion (197 girls and 140 boys, mean age $=12.4$ years). All children were biologically related to and currently living with the affected parent. Additional exclusion criteria included children with moderate-severe intellectual disability $(\mathrm{IQ}<50)$ and parents with bipolar disorder, mania/hypomania or psychosis at the time of interview. Two families were consequently excluded from waves 2 and 3 of the study because the depressed parent had been diagnosed as suffering from bipolar disorder since wave 1 assessment. Symptoms of psychiatric disorder in parents and offspring were separately assessed using age-appropriate standard research diagnostic interviews independently by two trained research psychologists on three occasions over the course of the study which began in April 2007 and finished in April 2011. The average time between the baseline and second assessment was 16.2 months and between the second and third assessment was 12.5 months. Further details on the sample characteristics and methodology have been described previously. ${ }^{9}$

For the main analyses, 30 families were excluded because they had not completed at least two waves of the data. A further 10 children were excluded from main analyses because they already met criteria for DSM-IV major depressive disorder at baseline and thus the blood pressure assessment did not precede the onset. Lastly 14 children were excluded because a measure of blood pressure and/or weight had not been completed at baseline either because there had been a fault in the equipment or the child had refused. This resulted in a final sample of 281 families (see figure 1 for more details). Psychiatric data were collected from parents and children through semistructured research diagnostic interviews and blood pressure and weight were assessed by the interviewer. Additional data on physical health problems, maternal education and social class were collected from parents and children from selfcompleted questionnaires that were mailed to the families 2 weeks before their interview.

Data were also utilised from a birth cohort study 'The Avon Longitudinal Study of Parents and Children (ALSPAC)' to allow replication of findings from the first high-risk sample. The cohort was set up to examine genetic and environmental determinants of health and development. ${ }^{10}$ The initial cohort consisted of 14062 children born to residents of the Bristol area, the UK, who had an expected date of delivery between 1 April 1991 and 31 December 1992 (http://www.alspac.bris.ac. uk). All pregnant women resident in three health districts in the old administrative county of Avon who had an estimated delivery between the above dates were eligible to participate. In addition, pregnant women who had migrated to the catchment area before the point of delivery were eligible. Recruitment was carried out by attempting to make contact with eligible women through ALSPAC staff visiting community locations and through using antenatal and maternity health services and media information to encourage contact and promote the study. ${ }^{10}$ The parents completed regular postal questionnaires concerning their child's health and development since birth. The children have completed questionnaires and attended annual assessment clinics since the age of 7 years. For analyses using the whole sample, 4830 children had data on blood pressure and weight at age 12 (2515 females and 2315 males; age range $11-14$ years; mean age $=12.8$ years) and depression at age 15 (age range $14-17$ years; mean age $=15.4$ years) Main replication analyses focused on the subsample of children whose mothers had experienced recurrent depression (at least two episodes); 612 children were included in these analyses (347 females and 265 males).

\section{MEASURES \\ The Early Prediction of Adolescent Depression (EPAD) Study}

Assessment of depression in the offspring of parents with recurrent depression: The Child and Adolescent Psychiatric Assessment (CAPA) is a semistructured research diagnostic interview that has high reliability and that is used to assess children's psychiatric symptoms. ${ }^{11}$ Parents are asked about their children's psychopathology and children are independently interviewed using the interview 




Figure 1 Flow chart of retention at each assessment in the Early Prediction of Adolescent Depression Study sample.

schedules (parent and child versions). The presence of any given symptom has to be rigorously assessed and only endorsed by the interviewer when it achieves the CAPA-defined symptom threshold. Interviewers were trained by the team who developed the CAPA and all interviews were recorded. Interview fidelity was checked through inter-rater reliability checks of 20 randomly selected recordings at each time point (10 parent report and 10 child report) and through weekly supervision by an academic clinician with extensive experience in using the interview. Average agreement between raters for DSM-IV psychiatric disorder was excellent $(\kappa=0.92)$, as was average agreement for depression symptoms $(\kappa=0.93)$. CAPA was used at each assessment and assesses the presence of a major depressive disorder in the child over the preceding 3 months. The parent and child versions were completed independently, with interviews conducted in separate rooms where possible and in $99 \%$ of cases, by separate researchers. Child diagnoses were generated using DSM-IV criteria, based on CAPA symptoms. The presence of a diagnosis was endorsed if either parent (reporting on the child) or child reported it as being present. Fidelity of the diagnostic algorithms was further checked as all those meeting diagnostic criteria and subthreshold cases were reviewed weekly by two senior clinical and academic child and adolescent 
psychiatrists. The total number of DSM-IV major depressive disorder symptoms was also computed from the CAPA.

New-onset major depressive disorder. The presence of a new-onset DSM-IV diagnosis of major depressive disorder at either the second or third assessment was defined by excluding children who had a baseline diagnosis of DSM-IV major depressive disorder.

Blood pressure: An Omron 705IT sphygmomanometer was used to measure blood pressure at each assessment while the child was in a seated position with their arm resting on a flat surface. A standard cuff was used to measure blood pressure in children aged 11 years and over and a small adult cuff was used for children under 11 years, unless overweight. Systolic and diastolic blood pressures were measured using standardised guidelines set out by the American Heart association. ${ }^{12}$ At least two readings were taken at $1 \mathrm{~min}$ apart using the right arm. When the difference between two readings was $5 \mathrm{~mm} \mathrm{Hg}$ or less an average was taken.

Weight and other potential confounders: Weight was considered to be a confounder of the relationship between blood pressure and depression due to its potential association with both. ${ }^{13}{ }^{14}$ Interviewers measured the weight of the children without shoes to the nearest $0.1 \mathrm{~kg}$ using Seca scales. We also examined whether the results were affected by the presence of physical health problems (parent reported), any medication use (child or parent reported) and using body mass index (BMI) instead of weight.

Demographics: The mother and father questionnaires completed at baseline were used to assess maternal education and highest parental social class. Maternal education was categorised according to whether the mother had completed higher education (A-levels, degree or postgraduate qualification). Parental social class was categorised according to whether either parent reported having a non-manual occupation.

\section{Avon Longitudinal Study of Parents and Children}

Maternal history of recurrent depression: Mothers completed regular questionnaires from pregnancy to when the child was aged 12 years including the questions "Have you had depression in the last year/last two years/since your child was born/ever?". The mother was also asked "Have you ever had severe depression?" on three occasions over this time period. Research diagnostic interview generated psychiatric data on parents are not available in ALSPAC. Recurrent depression in the replication data set thus had to be defined where mother had reported having depression on at least two separate occasions, and if at least one of these occasions was reported as being severe. These criteria were used to create a subsample that was as similar as possible to the primary high-risk sample.

Child depressive disorder. Parent reports on their child's symptoms were obtained using a structured diagnostic schedule (the Development and Wellbeing Assessment; DAWBA $^{15}$ ) that, like the CAPA, has been used widely for large-scale population studies. The parent-rated DAWBA was completed when the target age of the children was 13 years. Children were directly interviewed using the DAWBA at age 15 . The DAWBA assesses the presence of a depressive disorder over the preceding month. DSM-IV diagnoses of depression were generated at each time point using a well-defined computerised algorithm that predicts the likelihood of a clinical rater assigning each child a DSM-IV diagnosis of depression and generates diagnoses (see http://www.DAWBA.com for more information). A senior clinical psychiatrist reviewed the diagnoses and the DAWBA responses as part of the ALSPAC data collection process. ${ }^{16}$

Blood pressure. Systolic and diastolic blood pressures were measured at the clinic assessments when the target ages of the children were 12 and 15 years. Blood pressure was measured twice at each assessment with a Dinamap 9301 Vital Signs Monitor and a mean of both readings was taken.

Weight and other potential confounders: Interviewers measured the weight and height of the children in light clothing and without shoes at the clinic assessments when the target age of the children was 12 years. Weight was measured to the nearest $0.1 \mathrm{~kg}$ using Tanita scales (Tanita; Illinois, USA) and height was measured to the nearest $0.1 \mathrm{~cm}$ using a Harpenden stadiometer (Tarti; Turkey). BMI was then calculated $\left(\mathrm{kg} / \mathrm{m}^{2}\right)$. Maternal systolic blood pressure at 8 weeks of gestation was abstracted from antenatal medical records.

Demographics: The mother and father questionnaires completed during pregnancy were used to assess maternal education and highest parental social class. Maternal education was categorised according to whether the mother had completed higher education (A-levels or degree). Parental social class was categorised according to whether either parent reported having a non-manual occupation.

\section{Statistical methods}

Each set of analyses were conducted in two steps. First, descriptive statistics were examined in the high-risk sample and then in the replication cohort using the subsample of children with mothers that have experienced recurrent depression in the past. Next, regression analyses were performed to examine the association between blood pressure and depression in the high-risk sample and then in the subsample from the replication cohort. Logistic regression analyses were used when the dependent variable was dichotomous and ordinary least-squares linear regression analysis was used when the dependent variable was continuous. Continuous outcome data that were not normally distributed were transformed prior to analysis using a natural log transformation. Next, the linearity of the relationship between systolic blood pressure and future depressive disorder was examined by investigating the percentage of children with future depressive disorder by blood pressure quintiles, again in both samples. Next, receiver operating characteristic (ROC) analysis was performed 
to establish a cut-off for blood pressure in both samples that showed adequate sensitivity and specificity for detecting future depressive disorder. Lastly, the association between systolic blood pressure and future depressive disorder was investigated in the general population by using the entire ALSPAC sample and the presence of a multiplicative interaction between maternal depression and systolic blood pressure on future depressive disorder was examined. List-wise deletion was used to deal with missing data in all analyses and data were analysed using SPSS (V.20).

\section{RESULTS}

Demographic comparability of the EPAD high-risk sample (offspring of parents with recurrent depression), the ALSPAC replication subsample of children with mothers who have experienced recurrent depression and the whole ALSPAC sample

Table 1 shows that the three samples are comparable on a range of demographics (child age, gender, maternal education and parental social class).

\section{Descriptives from EPAD high-risk sample}

Mean systolic and diastolic blood pressures in the EPAD sample at baseline were 117.26 and $70.88 \mathrm{~mm} \mathrm{Hg}$ and SD were 13.18 and 11.39 , respectively. There were no significant differences between males and females for systolic or diastolic blood pressure at baseline. Systolic blood pressure at baseline was significantly correlated with age $(\mathrm{r}=0.23, \mathrm{p}<0.001)$, but diastolic blood pressure was not $(r=0.02, p=0.728)$. Systolic blood pressure was thus standardised for age and gender to create SD scores and all analyses for systolic blood pressure were run with the standardised variable. ${ }^{17}$ Mean blood pressures for each age group in this sample were generally higher than population norms. ${ }^{17} 18$ Systolic blood pressure at baseline was significantly associated with weight $(\mathrm{r}=0.25, \mathrm{p}<0.001)$, but diastolic blood pressure was not $(r=0.07, p=0.236)$. Given this finding all analyses with systolic blood pressure are reported controlling for weight.

Twenty-four children ( 5 boys and 19 girls) in the sample developed a new-onset depressive disorder (8.54\%).
Descriptives from ALSPAC data set-using the subsample of children with mothers who have experienced recurrent depression in the past

Mean systolic and diastolic blood pressures in this sample at age 12 were 111.10 and $56.61 \mathrm{~mm} \mathrm{Hg}$ and SD were 9.44 and 8.16 , respectively. There were no significant differences between males and females for systolic or diastolic blood pressure at age 12. Again, systolic blood pressure was standardised for age and gender to create SD scores and all analyses for systolic blood pressure were run with the standardised variable. The association between blood pressure and weight at age 12 was significant for systolic $(\mathrm{r}=0.45, \mathrm{p}<0.001)$ and diastolic blood pressures $(\mathrm{r}=0.18, \mathrm{p}<0.001)$, therefore all results are reported controlling for weight.

Eighteen children ( 5 boys and 13 girls) in the sample reported a depressive disorder over the last month at age $15(2.94 \%)$.

\section{Initial analyses in EPAD high-risk sample}

Logistic regression analyses were performed to investigate the association between blood pressure and newonset depressive disorder in the EPAD sample of children. It was found that as systolic blood pressure increased, risk for new-onset depressive disorder decreased, when adjusting for child's weight at baseline $(\mathrm{OR}=0.65,95 \%$ CI 0.44 to $0.96 ; \mathrm{p}=0.029)$, that is, lower systolic blood pressure at baseline significantly predicted new-onset depressive disorder. Diastolic blood pressure at baseline did not significantly predict the new-onset depressive disorder $(\mathrm{OR}=0.97,95 \%$ CI 0.92 to 1.01; $\mathrm{p}=0.120)$.

Results remained the same when the outcome was expanded to include the new-onset of more broadly defined mood-related diagnoses (primary diagnosis: major depressive disorder $n=22$, dysthymia $n=1$, cyclothymia $n=1$, bipolar disorder $n=3$, adjustment disorder with depressed $\operatorname{mood} n=4$ and depressive disorder not otherwise specified $n=5$ ). Results also remained similar when separately adjusting for medication use and physical health problems in the child and when adjusting for BMI instead of weight. The association was not significantly moderated by gender $(\mathrm{p}=0.769)$.

Table 1 Demographics at baseline in the EPAD sample, ALSPAC subsample of offspring of recurrently depressed mothers and the whole ALSPAC sample

$\operatorname{EPAD}(\mathrm{n}=281)$ of recurrently depressed mothers; $n=612)$

\begin{tabular}{llll}
\hline Child age: mean (SD) & $12.4(2.0)$ & $12.8(0.2)$ & $12.8(0.2)$ \\
Child gender (\% female) & 58.4 & 56.7 & 52.1 \\
Maternal education & 51.9 & 44.3 & 47.2
\end{tabular}

(\% higher education)

Parental social class

74.0

84.1

87.7

(\% non-manual)

ALSPAC, Avon Longitudinal Study of Parents and Children; EPAD, Early Prediction of Adolescent Depression study. 
Given few adolescents developed new-onset depressive disorder, the analysis was repeated using total depression symptom scores at wave 3 as the outcome. Linear regression analysis with systolic blood pressure at baseline as a predictor and depression symptoms at the wave 3 assessment as the outcome and again controlling for weight, showed significant association $(\beta=-0.13 ; \mathrm{p}=0.040)$.

To examine whether depressive disorder was a predictor of blood pressure, a linear regression analysis was performed with a diagnosis of depressive disorder at baseline as a predictor and systolic blood pressure at wave 3 assessment as the outcome, again controlling for child"s weight at baseline. This was non-significant $(\beta=-0.05 ; \mathrm{p}=0.412)$. Given the low number of individuals with depressive disorder at baseline the analysis was repeated using total depression symptoms at baseline as a predictor. Again the results were non-significant $(\beta=-0.07 ; p=0.286)$.

\section{Replication in ALSPAC data set-using the subsample of children with mothers who have experienced recurrent depression in the past}

Logistic regression analyses were undertaken in the replication sample. Again, it was found that as systolic blood pressure increased, risk for future depressive disorder decreased, when adjusting for child's weight at baseline $(\mathrm{OR}=0.48,95 \%$ CI 0.27 to $0.85 ; \mathrm{p}=0.012)$, that is, lower systolic blood pressure at age 12 significantly predicted depressive disorder at age 15. Diastolic blood pressure at age 12 did not significantly predict depressive disorder at age $15(\mathrm{OR}=0.98,95 \%$ CI 0.93 to $1.05 ; \mathrm{p}=0.597)$. Results remained similar when adjusting for BMI instead of weight and when additionally adjusting for maternal systolic blood pressure in pregnancy. The association was not significantly moderated by gender $(\mathrm{p}=0.102)$.

To examine whether depressive disorder was a predictor of blood pressure, a linear regression analyses was performed with a diagnosis of depressive disorder at age 13 as a predictor and systolic blood pressure at age 15 as the outcome, again controlling for child's weight. This was non-significant $(\beta=-0.04 ; p=0.378)$.

\section{Relationship between blood pressure and depressive}

\section{disorder in high-risk EPAD sample}

To further investigate the relationship between systolic blood pressure and depressive disorder in the EPAD sample, blood pressure was split into quintiles to examine the percentage of children with new-onset depressive disorder by blood pressure categories and the linearity of the relationship. As can be seen in figure 2, there is a lower risk of depressive disorder with higher levels of blood pressure, with the lowest quintile of systolic blood pressure having the highest percentage of depressive disorder cases.

\section{Replication in ALSPAC data set-using the subsample} of children with mothers who have experienced recurrent depression in the past

To further investigate the relationship between systolic blood pressure and depression in the replication sample,



Figure 2 Percentage of children with new-onset depressive disorder at follow-up by quintiles of systolic blood pressure at baseline in the Early Prediction of Adolescent Depression Study sample.

blood pressure was split into quintiles to examine the percentage of children with depression at age 15 by blood pressure categories and the linearity of the relationship.

As can be seen in figure 3, there is a lower risk of depressive disorder with higher levels of blood pressure, with the lowest quintile of systolic blood pressure having the highest percentage of depressive disorder cases.

Analyses so far have highlighted a significant association between lower systolic blood pressure and future depression in two different samples of offspring of parents with recurrent depression. Next, ROC analysis was performed to establish a cut-off for blood pressure in both samples that maximised sensitivity and specificity for detection of future depressive disorder. A cut-off point of below $0.025 \mathrm{SD}$ above the mean using standardised systolic blood pressure ${ }^{17}$ showed a sensitivity of $63 \%$ and a specificity of $66 \%$ for the high risk EPAD sample (this would approximately equate to systolic blood pressure below $112 \mathrm{~mm} \mathrm{Hg}$ for a 12-year-old boy and below $113 \mathrm{~mm} \mathrm{Hg}$ for a 12-year-old girl). A cut-off point of below 0.485 SD below the mean using standardised systolic blood pressure ${ }^{17}$ showed a sensitivity of

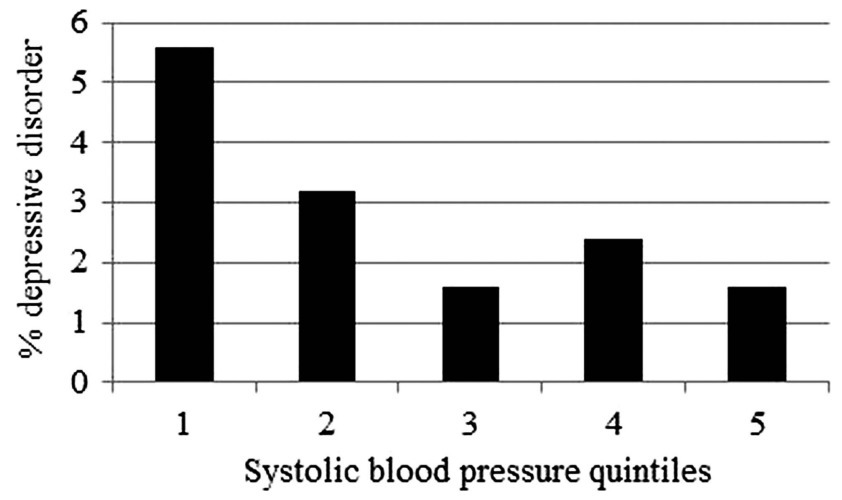

Figure 3 Percentage of children with depressive disorder at age 15 by quintiles of systolic blood pressure at age 12 in the Avon Longitudinal Study of Parents and Children (ALSPAC) sample. 
$61 \%$ and a specificity of $61 \%$ for the ALSPAC replication sample (this would approximately equate to systolic blood pressure below $108 \mathrm{~mm} \mathrm{Hg}$ for a 12-year-old boy or girl). Logistic regression analyses were performed to examine the strength of the association between low blood pressure and future depressive disorder using each of the cut-offs identified in each sample (table 2).

From these analyses it seems a cut-off for low systolic blood pressure for children aged 12 years lies between 108 and $113 \mathrm{~mm} \mathrm{Hg}$.

Lastly, supplementary analyses were performed to examine the association between blood pressure and future depression in the general population using the entire ALSPAC sample.

\section{Supplementary analyses using entire ALSPAC sample}

1 Testing the relationship between depressive disorder and blood pressure in the general population (not limiting analysis to those adolescents with a parental history of recurrent depression)

Descriptives: Mean systolic and diastolic blood pressures in the sample at age 12 were 111.01 and $56.53 \mathrm{~mm} \mathrm{Hg}$ and SD were 9.54 and 7.88 , respectively. There were no significant differences between males and females for systolic or diastolic blood pressure at age 12. Again, systolic blood pressure was standardised for age and gender to create SD scores and all analyses for systolic blood pressure were run with the standardised variable. The association between blood pressure and weight at age 12 was significant for systolic $(\mathrm{r}=0.44, \mathrm{p}<0.001)$ and diastolic blood pressures $(\mathrm{r}=0.19, \mathrm{p}<0.001)$, therefore all results are reported controlling for weight.

Seventy-five children (22 boys and 53 girls) in the sample reported a depressive disorder over the last month at age $15(1.55 \%)$.

Preliminary analyses: Logistic regression analyses were performed to investigate the association between blood pressure and depressive disorder. Systolic blood pressure at age 12 did not significantly predict depressive disorder at age $15(\mathrm{OR}=0.98,95 \%$ CI 0.76 to $1.27 ; \mathrm{p}=0.875)$. Diastolic blood pressure at age 12 did not significantly

Table 2 Association between low blood pressure and future depressive disorder in the EPAD and ALSPAC samples using the optimal cut-off for low blood pressure identified in each sample using ROC analysis

\begin{tabular}{|c|c|c|}
\hline & \multicolumn{2}{|l|}{ OR $(95 \% \mathrm{Cl})^{\star}$} \\
\hline & $\begin{array}{l}\text { Optimal cut-off } \\
\text { in EPAD sample } \\
(<0.025)\end{array}$ & $\begin{array}{l}\text { Optimal cut-off in } \\
\text { ALSPAC sample } \\
(<-0.485)\end{array}$ \\
\hline EPAD sample & $3.13(1.30,7.53)$ & $3.43(1.45,8.13)$ \\
\hline ALSPAC sample & $3.00(0.93,9.71)$ & $3.62(1.23,10.65)$ \\
\hline
\end{tabular}

predict depressive disorder at age $15(\mathrm{OR}=1.01,95 \%$ CI 0.98 to $1.04 ; \mathrm{p}=0.604$ ).

2 Examining whether a history of recurrent maternal depression moderated the relationship between systolic blood pressure and depression

Logistic regression analyses were then performed to test if recurrent maternal depression moderated the relationship between systolic blood pressure and depressive disorder. The predictor variables, systolic blood pressure and maternal recurrent depression were centred to convert them to their deviation form to remove nonessential multicollinearity. The interaction was found to be significant $(\mathrm{OR}=0.49,95 \%$ CI 0.27 to $0.89 ; \mathrm{p}=0.019$ ).

Systolic blood pressure was then categorised into 'high' and 'low' by splitting at the median, so that the interaction could be displayed graphically. As can be seen in figure 4, when recurrent maternal depression was present, children with low blood pressure showed the highest percentage of depressive disorder. However, when recurrent maternal depression was not present, children with high blood pressure showed the highest percentage of depressive disorder.

\section{DISCUSSION}

In this study we found that lower systolic blood pressure significantly predicted future new-onset depressive disorder among a sample of children and adolescents at high risk of developing depression because of a parental history of recurrent depression. This finding was replicated in a large community-based cohort study (ALSPAC) for children whose mothers reported a history of recurrent depression (replication sample). When investigating this relationship in more detail, it seemed that those with the lowest systolic blood pressure were most at risk of developing a depressive disorder in the future. A cut-off value for systolic blood pressure in 12-year-old children was identified as being within the range of 108 and $113 \mathrm{~mm} \mathrm{Hg}$. Finally when investigating this relationship in the entire population cohort (ALSPAC), the association between blood pressure and



Figure 4 Percentage of children with depressive disorder at age 15 within 'high' and 'low' systolic blood pressure groups at age 12 by maternal depression status in the Avon Longitudinal Study of Parents and Children (ALSPAC) sample. 
major depressive disorder was no longer significant. There was no evidence for an association in the opposite direction (depression predicting future blood pressure levels) either in the study sample or in the replication cohort nor was there an association between diastolic blood pressure and future depression in either data set.

In our study, while the average blood pressure in the offspring of parents with recurrent depressive disorder was slightly higher than population norms, it was those whose blood pressure was lower who were at most risk of developing depressive disorder. This was not true in adolescents from the general population. In adolescents from the general population who did not have a parent with recurrent depressive disorder, higher blood pressure showed some association with a higher risk of future depressive disorder. There have been no previously published longitudinal studies examining the relationship between blood pressure and depression in children from the general population or children at high risk of depression. In adults a 'vascular depression' hypothesis has been proposed to explain links between elevated blood pressure and depression ${ }^{19}$ but some adult studies have found cross-sectional links between low blood pressure and depression. ${ }^{20}{ }^{21}$ In addition, a study on elderly patients reported a fall in systolic blood pressure predicted the onset of depression. ${ }^{6}$ The aim in our study was however to look at links between blood pressure and depression prior to the usual age of the onset of hypertensive disease to examine developmental changes. Given the limited scientific literature on this topic, the mechanisms by which low blood pressure might precede depression for adolescent offspring of individuals with recurrent depressive disorder are unclear and we can at present only speculate as to what these might be. Possible mechanisms include shared risks (genetic and/or environmental) that contribute to lower blood pressure and depression that are especially enriched in those offspring most at risk of developing depressive disorder in the near future. Another possibility is that low blood pressure, in those who are familially/genetically vulnerable to depression, represents an early manifestation (possibly through autonomic system dysregulation) or prodromal phase of major depressive disorder. There is strikingly limited research on biological links between early mental health problems and physical health as well as on autonomic system function in young people who are familially vulnerable to depression. ${ }^{22}$ Our findings highlight the need for further research on links between mental and physical health in young people.

\section{Strengths and limitations}

This is the first study we are aware of to report on the longitudinal relationship between blood pressure and depressive disorder in adolescents, an important period for the onset of depression. In the main sample, children and adolescents were followed up at three points over a 4 -year period with a high retention rate of over $80 \%$. A similar pattern of results was found in a large communitybased cohort study (ALSPAC) for those children of mothers with a history of recurrent depression. In both samples, diagnoses were systematically ascertained using interview and blood pressure readings were measured according to standardised protocol. In addition, potential confounders of the relationship were taken into account. There were also possible limitations of the study. Blood pressures were measured using an electronic device which uses an oscillometric technique rather than the auscultatory technique that most population norms are based on and it has been noted that these readings are not equivalent. However, there is a lack of consensus as to whether using different methods leads to any systematic bias and inaccuracies seem more related to not using a standardised technique rather than the instrument. ${ }^{23}$ In addition, the cut-off values identified in the high risk and replication samples differed slightly. This may have been because of differences in measurement techniques in the two studies. These results need to be replicated in other samples in order to establish a more precise cut-off for low systolic blood pressure. There was also relatively a high false-positive rate for the cut-offs identified in both the high risk and the replication sample (34\% and 39\%, respectively). However, the aim of this analysis was not to develop a screening tool for depression but to use ROC analysis as a method to maximise sensitivity and specificity in determining the optimal cut-off value for low blood pressure. Moreover, some of the individuals labelled as 'false positives' will have subthreshold depression which has been found to be associated with impairment and to predict escalation to future disorder. ${ }^{24}{ }^{25}$ Despite being a high-risk sample, the number of children with depressive disorder was small and many of the sample had not been though the age of maximum risk for developing a depressive disorder so this could lead to an underestimate of the effects of risk factors. In addition, the number of children with depressive disorder was also low in the replication sample, partly because only a self-report measure of depressive disorder was available at age 15 and partly because of selective attrition over time. Previous studies have reported that although attrition has affected prevalence rates of depression in the mother and internalising disorders in the children, the associations between risks and outcomes remained intact, although conservative estimates of the likely true effects. ${ }^{26}{ }^{27}$ Lastly, the subsample of recurrently depressed mothers in the replication data set is likely to index a less severe group as maternal selfreport of depression was used as opposed to defining episodes of depression using DSM-IV criteria as was performed in the main data set.

In summary, in our study of adolescents at high risk of depression we found that low blood pressure was associated with major depressive disorder. This finding was replicated in an independent cohort. Future research is needed using different populations to confirm this relationship as it is a novel finding and to investigate the mechanisms by which the relationship between low 
blood pressure and depressive disorder in children at risk for depression may arise.

Acknowledgements The authors are extremely grateful to all the families who took part in the Early Prediction of Adolescent Depression (EPAD) study, the general practice surgeries for their help with recruiting them and the whole EPAD team. They also thank the other investigators involved in the original EPAD study, Dr Robert Potter, Dr Stephan Collishaw, Dr Daniel Smith, Professor Michael Owen, Dr Frances Rice and Professor Nick Craddock. In addition, we are also extremely grateful to all the families who took part in the Avon Longitudinal Study of Parents and Children (ALSPAC) study, the midwives for their help with recruiting them and the whole ALSPAC team, which includes interviewers, computer and laboratory technicians, clerical workers, research scientists, volunteers, managers, receptionists and nurses. The UK Medical Research Council and the Wellcome Trust (Grant ref: 092731) and the University of Bristol provide core support for ALSPAC

Contributors GeH jointly designed the paper and the analysis, carried out the analysis, jointly drafted and revised the paper, AnT helped with initial conception of the study and with study design and critically revised the draft for important intellectual content, GoH advised on analysis in the paper and critically revised the paper, AjT was responsible for conception of the paper, jointly designed the study and analysis, joint initial drafting and revising the paper. All authors approved the final version prior to submission of the paper. $\mathrm{AjT}$ is the guarantor.

Funding Initial funding for this study was provided by the British Medical Association (Strutt and Harper) Grant and the Sir Jules Thorn Charitable Trust (Grant ref: 06/JTA). The National Institute for Social Care and Health Research Academic Health Science Collaboration (AHSC) fellowship provided funding for one of the authors (AKT). The funders had no further role in the study design, the collection, analysis and interpretation of data, the writing of the report, or in the decision to submit the paper for publication.

Competing interests None.

Ethics approval South Wales Multi-centre Research Ethics Committee (MREC), the ALSPAC Ethics and Law committee and the Local Research Ethics Committees.

Provenance and peer review Not commissioned; externally peer reviewed.

Data sharing statement No additional data are available.

Open Access This is an Open Access article distributed in accordance with the Creative Commons Attribution Non Commercial (CC BY-NC 3.0) license, which permits others to distribute, remix, adapt, build upon this work noncommercially, and license their derivative works on different terms, provided the original work is properly cited and the use is non-commercial. See: http:// creativecommons.org/licenses/by-nc/3.0/

\section{REFERENCES}

1. Evans DL, Charney DS, Lewis L, et al. Mood disorders in the medically ill: scientific review and recommendations. Biol Psychiatry 2005;58:175-89.

2. Davidson K, Jonas BS, Dixon KE, et al. Do depression symptoms predict early hypertension incidence in young adults in the CARDIA study? Coronary Artery Risk Development in Young Adults. Arch Intern Med 2000;160:1495-500.

3. Licht CM, de Gaus EJC, Seldenrijk A, et al. Depression is associated with decreased blood pressure, but antidepressant use increases the risk for hypertension. Hypertension 2009;53:631-8.

4. Patten SB, Williams JV, Lavorato $\mathrm{DH}$, et al. Major depression as a risk factor for high blood pressure: epidemiologic evidence from a national longitudinal study. Psychosom Med 2009;71:273-9.
5. Hildrum B, Romild U, Holmen J. Anxiety and depression lowers blood pressure: 22-year follow-up of the population based HUNT study, Norway. BMC Public Health 2011;11:601.

6. Paterniti S, Verdier-Taillefer $\mathrm{MH}$, Geneste $\mathrm{C}$, et al. Low blood pressure and risk of depression in the elderly-A prospective community-based study. Br J Psychiatry 2000;176:464-7.

7. Doris A, Ebmeier K, Shajahan P. Depressive illness. Lancet 1999;354:1369-75.

8. Berenson GS. Cardiovascular risk begins in childhood: a time for action. Am J Prev Med 2009;37(1 Suppl):S1-2.

9. Mars B, Collishaw S, Smith D, et al. Offspring of parents with recurrent depression: which features of parent depression index risk for offspring psychopathology? J Affect Disord 2012;136:44-53.

10. Boyd A, Golding J, Macleod J, et al. Cohort profile: the 'Children of the 90s'-the index offspring of the Avon Longitudinal Study of Parents and Children. Int J Epidemiol 2012;42:111-27.

11. Angold A, Costello EJ. The Child and Adolescent Psychiatric Assessment (CAPA). J Am Acad Child Adolesc Psychiatry 2000;39:39-48

12. Smith L. New AHA recommendations for blood pressure measurement. Am Fam Physician 2005;72:1391-8.

13. Anderson SE, Cohen P, Naumova EN, et al. Adolescent obesity and risk for subsequent major depressive disorder and anxiety disorder: prospective evidence. Psychosom Med 2007;69:740-7

14. Bekkers MB, Brunekreef B, Koppelman GH, et al. BMI and waist circumference; cross-sectional and prospective associations with blood pressure and cholesterol in 12-year-olds. PLOS ONE 2012;7: e51801.

15. Goodman R, Ford T, Richards $\mathrm{H}$, et al. The Development and Well-Being Assessment: description and initial validation of an integrated assessment of child and adolescent psychopathology. J Child Psychol Psychiatry 2000;41:645-55.

16. Goodman A, Heiervang E, Collishaw S, et al. The 'DAWBA bands' as an ordered-categorical measure of child mental health: description and validation in British and Norwegian samples. Soc Psychiatry Psychiatr Epidemiol 2011;46:521-32.

17. Jackson LV, Thalange NK, Cole TJ. Blood pressure centiles for Great Britain. Arch Dis Child 2007;92:298-303.

18. National High Blood Pressure Education Program Working Group on High Blood Pressure in Children and Adolescents. Fourth report on the diagnosis, evaluation, and treatment of high blood pressure in children and adolescents. Pediatrics 2004;114:555-76.

19. Taylor WD, Aizenstein HJ, Alexopoulos GS. The vascular depression hypothesis: mechanisms linking vascular disease with depression. Mol Psychiatry 2013;18:963-74.

20. Hildrum B, Mykletun A, Stordal E, et al. Association of low blood pressure with anxiety and depression: the Nord-Trøndelag Health Study. J Epidemiol Community Health 2007;61:53-8.

21. Moldin SO, Scheftner WA, Rice JP, et al. Association between major depressive disorder and physical illness. Psychol Med 1993;23:755-61.

22. McGirr A, Diaconu G, Berlim MT, et al. Dysregulation of the sympathetic nervous system, hypothalamic-pituitary-adrenal axis and executive function in individuals at risk for suicide. $J$ Psychiatry Neurosci 2010;35:399-408.

23. Portman RJ, Sorof JM, Ingelfinger JR. Pediatric hypertension. Humana Press Inc, 2004.

24. Fergusson D, Horwood LJ, Ridder EM, et al. Subthreshold depression in adolescence and mental health outcomes in adulthood. Arch Gen Psychiatry 2005;62:66-72.

25. Johnson JG, Cohen P, Kasen S. Minor depression during adolescence and mental health outcomes during adulthood. $\mathrm{Br} \mathrm{J}$ Psychiatry 2009;195:264-5.

26. Barker ED, Copeland W, Maughan B, et al. Relative impact of maternal depression and associated risk factors on offspring psychopathology. Br J Psychiatry 2012;200:124-9.

27. Wolke D, Waylen A, Samara M, et al. Selective drop-out in longitudinal studies and non-biased prediction of behaviour disorders. Br J Psychiatry 2009;195:249-56. 